JouRnal OF Universal MATHEMATICS

Vol.2 No.2 PP.137-143 (2019)

ISSN-2618-5660

\title{
SOME COMPUTATIONS BETWEEN SUMS OF POWERS OF CONSECUTIVE INTEGERS AND ALTERNATING SUMS OF POWERS OF CONSECUTIVE INTEGERS
}

\author{
UGUR DURAN $^{1, *}$ AND MEHMET ACIKGOZ ${ }^{2}$
}

\begin{abstract}
We study on the sums of powers of consecutive integers and alternating sums of power of consecutive integers. We derive many identities and correlations including Bernoulli, Euler and Genocchi polynomials and numbers.
\end{abstract}

\section{INTRODUCTION}

For a long time, Bernoulli, Euler and Genocchi polynomials and numbers and its generalizations have been extensively studied and investigated by many mathematicians and physicists (see [1-7]). Acikgoz et al. [?] considered the evaluation of the sum of more general series by Bernstein polynomials. Araci et al. [?] gave several novel identities for product of two Genocchi polynomials including Euler polynomials and Bernoulli polynomials and derived some applications for Genocchi polynomials to study a matrix formulation. Cheon [?] obtained a simple property of the Bernoulli polynomials and the Euler polynomials and also the relationship between two polynomials is derived. El-Makkawy et al. [?] provided a new approach in order to derive relations and identities for Bernoulli, Euler and Genocchi polynomials and numbers by means of the series manupulation prosedure. Kim [?] developed a formula for alternating sums of powers of consequtive integers by means of the Euler numbers. Shen [?] gave a formula for the sums of powers of consequtive integers by means of the Bernoulli numbers.

In the complex plane, the Bernoulli polynomials $B_{n}(x)(n \geq 0)$ are defined via the following Taylor series expansion about $z=0$ :

$$
\sum_{n=0}^{\infty} B_{n}(x) \frac{z^{n}}{n !}=\frac{z}{e^{z}-1} e^{x z} \quad(|z|<2 \pi),
$$

from which one can obtain the Bernoulli numbers as values $B_{n}(0):=B_{n}$ that also can be generated by the following generating functions

$$
\sum_{n=0}^{\infty} B_{n} \frac{z^{n}}{n !}=\frac{z}{e^{z}-1} \quad(|z|<2 \pi)
$$

Corresponding Author*

2010 Mathematics Subject Classification- 11B68, 11B83, 81S40.

Keywords and Phrases- Euler numbers and polynomials, Bernoulli numbers and polynomials, Genocchi numbers and polynomials, power sums. 
see [1-7] and references cited therein. The well known relation between the Bernoulli numbers and polynomials is obtained directly by (1) and (2) as

$$
B_{n}(x)=\sum_{k=0}^{n}\left(\begin{array}{l}
n \\
k
\end{array}\right) B_{n-k} x^{k} .
$$

The first few Bernoulli numbers are $1,-\frac{1}{2}, \frac{1}{6}, 0,-\frac{1}{30}, 0, \frac{1}{42}(n \geq 0)$ and the first few Bernoulli polynomials are $1, x-\frac{1}{2}, x^{2}-x+\frac{1}{6}, x^{3}-\frac{3}{2} x^{2}+\frac{1}{2} x, x^{4}-x^{3}+x^{2}-\frac{1}{30}$, $x^{5}-\frac{5}{2} x^{4}+\frac{5}{3} x^{3}-\frac{1}{6} x, x^{6}-3 x^{5}-\frac{5}{2} x^{4}+\frac{1}{2} x^{2}+\frac{1}{42}(n \geq 0)$.

The Euler polynomials $E_{n}(x)(n \geq 0)$ are defined via the Appell sequence with $g(z)=\frac{1}{2}\left(e^{z}+1\right)$ with the corresponding generating function

$$
\sum_{n=0}^{\infty} E_{n}(x) \frac{z^{n}}{n !}=\frac{2}{e^{z}+1} e^{x z} \quad(|z|<\pi) .
$$

Also, the Euler numbers $E_{n}(0):=E_{n}$ are given by the following Taylor series expansion at $z=0$ :

$$
\sum_{n=0}^{\infty} E_{n} \frac{z^{n}}{n !}=\frac{2}{e^{z}+1} \quad(|z|<\pi)
$$

see $[2,3,5,6]$ and references cited therein. The familiar formula for the Euler numbers and polynomials is directly attained by (4) and (5) as

$$
E_{n}(x)=\sum_{k=0}^{n}\left(\begin{array}{l}
n \\
k
\end{array}\right) E_{k} x^{n-k} .
$$

The first few Euler numbers are 1, $-\frac{1}{2}, 0, \frac{1}{4}, 0,-\frac{1}{2}, 0(n \geq 0)$ and the first few Euler polynomials are $1, x-\frac{1}{2}, x^{2}-x, x^{3}-\frac{3}{2} x^{2}+\frac{1}{4}, x^{4}-2 x^{3}+x, x^{5}-\frac{5}{2} x^{4}+\frac{5}{2} x^{2}-\frac{1}{2}$, $x^{6}-3 x^{5}+5 x^{3}-3 x(n \geq 0)$.

The relation between Bernoulli and Euler polynomials is given by the following formula

$$
B_{n}(x)=\sum_{\substack{k=0 \\
k \neq 1}}\left(\begin{array}{l}
n \\
k
\end{array}\right) B_{k} E_{n-k}(x) \quad \text { see [?]. }
$$

The Genocchi polynomials $G_{n}(x)(n \geq 0)$ are defined by the following power series expansion about $z=0$ :

$$
\sum_{n=0}^{\infty} G_{n}(x) \frac{z^{n}}{n !}=\frac{2 z}{e^{z}+1} e^{x z} \quad(|z|<\pi) .
$$

Then, one can obtain the Genocchi numbers as values $G_{n}(0):=G_{n}$ that also can be generated by the following generating functions

$$
\sum_{n=0}^{\infty} G_{n} \frac{z^{n}}{n !}=\frac{2 z}{e^{z}+1} \quad(|z|<\pi)
$$

see $[2,5]$ and references cited therein. The well known relation between the Genocchi numbers and polynomials is readily derived from (7) and (8) as

$$
G_{n}(x)=\sum_{k=0}^{n}\left(\begin{array}{l}
n \\
k
\end{array}\right) G_{n-k} x^{k} .
$$


The first few Euler numbers are $0,1,-1,0,1,-3,0(n \geq 0)$ and the first few Euler polynomials are $0,1,2 x-1,3 x^{2}-3 x, 4 x^{3}-6 x^{2}+1,5 x^{4}-10 x^{3}+5 x$, $6 x^{5}-15 x^{4}+15 x^{2}-3(n \geq 0)$.

The relations between the Euler and Genocchi polynomials and numbers are given by

$$
E_{n}(x)=\frac{G_{n+1}(x)}{n+1} \text { and } E_{n}=\frac{G_{n+1}}{n+1} \quad(n>0) .
$$

For non-negative integers $k>1, n$, let $S_{n}(k)$ the sums of the $n$-th powers of positive integers up to $k-1$ such that

$$
S_{n}(k)=\sum_{l=0}^{k-1} l^{n}=1^{n}+2^{n}+\cdots+(k-1)^{n} .
$$

Some special cases are given below:

$$
\begin{aligned}
S_{1}(k) & =\frac{1}{2} k^{2}-\frac{1}{2} k, \\
S_{2}(k) & =\frac{1}{3} k^{3}-\frac{1}{2} k^{2}+\frac{1}{6} k, \\
S_{3}(k) & =\frac{1}{4} k^{4}-\frac{1}{2} k^{3}+\frac{1}{4} k .
\end{aligned}
$$

Note that $S_{n}(k)$ is a polynomial in $k$ of the form

$$
S_{n}(k)=\frac{1}{n+1} k^{n+1}-\frac{1}{2} k^{n}+a_{n-1} k^{n-1}+\cdots+a_{1} k
$$

and satisfy

$$
\frac{d}{d k} S_{n}(k)=k^{n}-\frac{n}{2} k^{n-1}+\cdots .
$$

Jacques Bernoulli (1654-1705) gave the following result:

There exists a unique monic polynomial of degree $n$, say $B_{n}(x)$ (called the Bernoulli polynomials given in (1)), such that

$$
\begin{aligned}
S_{n}(k) & =1^{n}+2^{n}+\cdots+(k-1)^{n}=\int_{0}^{k} B_{n}(x) d x \\
& =\frac{B_{n+1}(k)-B_{n+1}(0)}{n+1}
\end{aligned}
$$

which is proved by Shen [?] in 2003.

A relationship between $S_{n}(k)$ and $B_{n}(x)$ is given as

$$
S_{n}(k)=\frac{1}{n+1} \sum_{l=0}^{n}\left(\begin{array}{c}
n+1 \\
l
\end{array}\right) B_{l} k^{n+1-l} .
$$

Let $n, k$ be positive integers with $k>1$. The alternating sums $T_{n}(k)$ of the $n$-th powers of positive integers up to $k-1$ :

$$
T_{n}(k)=\sum_{l=0}^{k-1}(-1)^{l} l^{n}=-1+2^{n}-\cdots+(-1)^{k-1}(k-1)^{n} .
$$

Kim [?] gave the following relation for $T_{n}(k)$ :

$$
T_{n}(k)=\frac{(-1)^{k+1}}{2} \sum_{l=0}^{n-1}\left(\begin{array}{l}
n \\
l
\end{array}\right) E_{l} k^{n-l}+\frac{E_{n}}{2}\left(1+(-1)^{k+1}\right) .
$$


If $k \equiv 0(\bmod 2)$, then it becomes

$$
T_{n}(k)=-\frac{1}{2} \sum_{l=0}^{n-1}\left(\begin{array}{l}
n \\
l
\end{array}\right) E_{l} k^{n-l} .
$$

If $k \equiv 1(\bmod 2)$, it reduces to following relation

$$
T_{n}(k)=\frac{1}{2} \sum_{l=0}^{n-1}\left(\begin{array}{l}
n \\
l
\end{array}\right) E_{l} k^{n-l}+E_{n}
$$

\section{Main Results}

In this part, we obtain some correlations including Bernoulli, Euler and Genocchi polynomials and numbers by using the properties of the $S_{n}(k)$ and $T_{n}(k)$.

In view of (11) and (12), for $k>1$, we see that

$$
\begin{aligned}
S_{n}(k)+T_{n}(k) & =\sum_{l=0}^{k-1} l^{n}+\sum_{l=0}^{k-1}(-1)^{l} l^{n} \\
& =1^{n}+2^{n}+\cdots+(k-1)^{n}+-1^{n}+2^{n}-\cdots+(-1)^{k-1}(k-1)^{n} \\
& =2\left(2^{n}+4^{n}+\left(-1+(-1)^{k-2}\right)(k-2)^{n}+\left(1+(-1)^{k-1}\right)(k-1)^{n}\right) \\
& =\left\{. 2 ^ { n + 1 } ( 1 ^ { n } + 2 ^ { n } + \cdots + ( \frac { k - 1 } { 2 } ) ^ { n } ) \text { if } k \text { is an odd integer, } 2 ^ { n + 1 } \left(1^{n}+2^{n}+\cdots+\left(\frac{k-2}{2}\right.\right.\right.
\end{aligned}
$$

Hence, we obtain that

(15)

$\frac{S_{n}(k)+T_{n}(k)}{2^{n+1}}=\left\{. S_{n}\left(\frac{k+1}{2}\right)\right.$ if $k$ is an odd integer, $S_{n}\left(\frac{k}{2}\right)$ if $k$ is an even integer.

Let $k>1$ be an odd integer. By (11), (12) and (15), we have

$$
\begin{aligned}
\frac{S_{n}(k)+T_{n}(k)}{2^{n+1}} & =\frac{1}{2^{n+1}}\left[\frac{1}{n+1} \sum_{l=0}^{n}\left(\begin{array}{c}
n+1 \\
l
\end{array}\right) B_{l} k^{n+1-l}+\frac{1}{2} \sum_{l=0}^{n-1}\left(\begin{array}{c}
n \\
l
\end{array}\right) E_{l} k^{n-l}+E_{n}\right] \\
(16) & =\frac{1}{2^{n+1}}\left[\sum_{l=0}^{n}\left(\begin{array}{c}
n \\
l
\end{array}\right) \frac{B_{l} k^{n+1-l}}{n+1-l}+\frac{1}{2}\left(E_{n}(k)+E_{n}\right)\right]
\end{aligned}
$$

and

$$
S_{n}\left(\frac{k+1}{2}\right)=\sum_{l=0}^{n}\left(\begin{array}{l}
n \\
l
\end{array}\right) \frac{B_{l}}{n+1-l}\left(\frac{k+1}{2}\right)^{n+1-l} .
$$

Thus, from (6), (16) and (17), we state the following theorem.

The following correlation including Bernoulli numbers and Euler numbers and polynomials

$$
E_{n}(k)+E_{n}=\sum_{l=0}^{n}\left(\begin{array}{l}
n \\
l
\end{array}\right) \frac{2 B_{l}}{n+1-l}\left(2^{l}(k+1)^{n+1-l}-k^{n+1-l}\right)
$$

holds true for $k>1$ being odd integer.

In view of (10) and (18), the following result holds true for $k>1$ being odd integer. 
The relation between Bernoulli and Genocchi numbers and polynomials holds true for $k>1$ :

$$
G_{n+1}(k)+G_{n+1}=\sum_{l=0}^{n}\left(\begin{array}{c}
n+1 \\
l
\end{array}\right) 2 B_{l}\left(2^{l}(k+1)^{n+1-l}-k^{n+1-l}\right) .
$$

Let $k>1$ be an even positive integer. In view of (11), (12) and (15), we have

$$
\begin{aligned}
\frac{S_{n}(k)+T_{n}(k)}{2^{n+1}} & =\frac{1}{2^{n+1}}\left[\sum_{l=0}^{n}\left(\begin{array}{l}
n \\
l
\end{array}\right) \frac{B_{l} k^{n+1-l}}{n+1-l}-\frac{1}{2} \sum_{l=0}^{n-1}\left(\begin{array}{c}
n \\
l
\end{array}\right) E_{l} k^{n-l}\right] \\
& =\frac{1}{2^{n+1}}\left[\sum_{l=0}^{n}\left(\begin{array}{l}
n \\
l
\end{array}\right) \frac{B_{l} k^{n+1-l}}{n+1-l}-\frac{1}{2} \sum_{l=0}^{n}\left(\begin{array}{c}
n \\
l
\end{array}\right) E_{l} k^{n-l}+\frac{E_{n}}{2}\right] \\
& =\frac{1}{2^{n+1}}\left[\sum_{l=0}^{n}\left(\begin{array}{l}
n \\
l
\end{array}\right) \frac{B_{l} k^{n+1-l}}{n+1-l}-\frac{1}{2}\left(E_{n}(k)-E_{n}\right)\right]
\end{aligned}
$$

and

$$
S_{n}\left(\frac{k}{2}\right)=\sum_{l=0}^{n}\left(\begin{array}{l}
n \\
l
\end{array}\right) \frac{B_{l}}{n+1-l}\left(\frac{k}{2}\right)^{n+1-l} .
$$

Hence, by (??) and (??), we state the following theorem.

The following correlation including Bernoulli numbers and Euler numbers and polynomials

$$
E_{n}(k)-E_{n}=\sum_{l=0}^{n}\left(\begin{array}{l}
n \\
l
\end{array}\right) \frac{2 k^{n+1-l} B_{l}}{n+1-l}\left(1-2^{l}\right)
$$

holds true for $k>1$ being even integer.

In view of (10) and (??), the following result holds true for $k>1$ being even integer.

The following relation is valid for $k>1$ being even integer

$$
G_{n+1}(k)-G_{n+1}=\sum_{l=0}^{n}\left(\begin{array}{c}
n+1 \\
l
\end{array}\right) 2 k^{n+1-l} B_{l}\left(1-2^{l}\right) .
$$

The following correlations holds true (see [?]):

$$
\begin{gathered}
G_{n}=2\left(1-2^{n}\right) B_{n} \quad n \geq 0 \\
G_{n}=\sum_{l=0}^{n-1}\left(\begin{array}{l}
n \\
l
\end{array}\right) 2^{l} B_{l} \quad(n \geq 1) \\
G_{n}(x)=-2 \sum_{l=1}^{n-1}\left(\begin{array}{l}
n \\
l
\end{array}\right) E_{l} B_{n-l}(x) \quad(n \geq 1) .
\end{gathered}
$$

We now provide some correlations and identities for Bernoulli and Euler polynomials and numbers via subsequent theorems.

Let $k>1$ be an odd integer and $n \geq 0$. We then obtain

$$
\sum_{l=1}^{n}\left(\begin{array}{c}
n+1 \\
l
\end{array}\right) E_{l} B_{n+1-l}(k)+\left(2^{n+1}-1\right) B_{n+1}=\sum_{l=0}^{n}\left(\begin{array}{c}
n+1 \\
l
\end{array}\right) B_{l}\left(k^{n+1-l}-2^{l}(k+1)^{n+1-l}\right) .
$$


Proof. From (??), (??) and (??), we observe that

$$
\begin{aligned}
G_{n+1}(k)+G_{n+1} & =-2 \sum_{l=1}^{n}\left(\begin{array}{c}
n+1 \\
l
\end{array}\right) E_{l} B_{n+1-l}(k)+2\left(1-2^{n+1}\right) B_{n+1} \\
& =\sum_{l=0}^{n}\left(\begin{array}{c}
n+1 \\
l
\end{array}\right) 2 B_{l}\left(2^{l}(k+1)^{n+1-l}-k^{n+1-l}\right),
\end{aligned}
$$

which yields to the desired result (??).

Let $k>1$ be an odd integer and $n \geq 1$. We then attain

$$
\sum_{l=1}^{n}\left(\begin{array}{c}
n+1 \\
l
\end{array}\right) E_{l} B_{n+1-l}(k)-\sum_{l=0}^{n}\left(\begin{array}{c}
n+1 \\
l
\end{array}\right) 2^{l-1} B_{l}=\sum_{l=0}^{n}\left(\begin{array}{c}
n+1 \\
l
\end{array}\right) B_{l}\left(k^{n+1-l}-2^{l}(k+1)^{n+1-l}\right) .
$$

Proof. By means of (??), (??) and (??), we see that

$$
\begin{aligned}
G_{n+1}(k)+G_{n+1} & =-2 \sum_{l=1}^{n}\left(\begin{array}{c}
n+1 \\
l
\end{array}\right) E_{l} B_{n+1-l}(k)+\sum_{l=0}^{n}\left(\begin{array}{c}
n+1 \\
l
\end{array}\right) 2^{l} B_{l} \\
& =\sum_{l=0}^{n}\left(\begin{array}{c}
n+1 \\
l
\end{array}\right) 2 B_{l}\left(2^{l}(k+1)^{n+1-l}-k^{n+1-l}\right),
\end{aligned}
$$

which gives the asserted result (??).

Let $k>1$ be an even integer and $n \geq 0$. We then acquire (29)

$$
\sum_{l=1}^{n}\left(\begin{array}{c}
n+1 \\
l
\end{array}\right) E_{l} B_{n+1-l}(k)+\left(1-2^{n+1}\right) B_{n+1}=\sum_{l=0}^{n}\left(\begin{array}{c}
n+1 \\
l
\end{array}\right) k^{n+1-l} B_{l}\left(1-2^{l}\right) .
$$

Proof. In view of (??), (??) and (??), we get

$$
\begin{aligned}
G_{n+1}(k)-G_{n+1} & =-2 \sum_{l=1}^{n}\left(\begin{array}{c}
n+1 \\
l
\end{array}\right) E_{l} B_{n+1-l}(k)-2\left(1-2^{n+1}\right) B_{n+1} \\
& =\sum_{l=0}^{n}\left(\begin{array}{c}
n+1 \\
l
\end{array}\right) 2 k^{n+1-l} B_{l}\left(1-2^{l}\right),
\end{aligned}
$$

which implies the desired result (??).

Let $k>1$ be an even integer and $n \geq 1$. We then get (30)

$$
\sum_{l=1}^{n}\left(\begin{array}{c}
n+1 \\
l
\end{array}\right) E_{l} B_{n+1-l}(k)+\sum_{l=0}^{n}\left(\begin{array}{c}
n+1 \\
l
\end{array}\right) 2^{l-1} B_{l}=\sum_{l=0}^{n}\left(\begin{array}{c}
n+1 \\
l
\end{array}\right) k^{n+1-l} B_{l}\left(2^{l}-1\right) .
$$

Proof. The proof of this theorem just follows from (??), (??) and (??).

$$
\begin{aligned}
G_{n+1}(k)-G_{n+1} & =-2 \sum_{l=1}^{n}\left(\begin{array}{c}
n+1 \\
l
\end{array}\right) E_{l} B_{n+1-l}(k)-\sum_{l=0}^{n}\left(\begin{array}{c}
n+1 \\
l
\end{array}\right) 2^{l} B_{l} \\
& =\sum_{l=0}^{n}\left(\begin{array}{c}
n+1 \\
l
\end{array}\right) 2 k^{n+1-l} B_{l}\left(1-2^{l}\right),
\end{aligned}
$$

which directly provided the result (??). 


\section{Conclusion}

In the present paper, the sums of powers of consecutive integers and alternating sums of power of consecutive integers have been studied and several relations and formulas including Bernoulli, Euler and Genocchi polynomials and numbers have been derived.

The first author is partly supported by the TÜBİTAK-BIDEB Scholarship Programme.

\section{REFERENCES}

[1] Acikgoz, M., Koca, I., Araci, S. The evaluation of the sums of more general series by Bernstein polynomials, Journal of Analysis and Number Theory, 4(1) (2016), 71-74.

[2] Araci, S., Acikgoz, M., Sen, E. Some new formulae for Genocchi numbers and polynomials involving Bernoulli and Euler polynomials, International Journal of Mathematics and Mathematical Sciences, Vol. 2014 (2014), Article ID 760613, 7 pages. doi:10.1155/2014/760613

[3] Cheon, G.-S. A note on the Bernoulli and Euler polynomials, Applied Mathematics Letters, 16(3) (2003), 365-368.

[4] Coen, L. E. S. Sums of Powers and the Bernoulli Numbers, Master Thesis, Eastern Illinois University, 1996.

[5] E.-Mikkawy, M., Atlan, F. Derivation of identities involving some special polynomials and numbers via generating functions with applications, Applied Mathematics and Computation, 220 (2013), 518-535.

[6] Kim, T. A note on the alternating sums of powers of consecutive integers, Proceedings of the Jangjeon Mathematical Society, 8(2) (2005), 175-178.

[7] Shen, Y.-Y. A note on the sums of powers of consecutive integers, Tunghai Science, 5 (2005), 101-106.

${ }^{1}$ Department of the Basic Concepts of Engineering, Faculty of Engineering and Natural Sciences, İskenderun Technical University, TR-31200, Hatay, Turkey

Email address: mtdrnugur@gmail. $\mathrm{com}^{1}$

${ }^{2}$ Department of Mathematics, Faculty of Arts and Sciences, Gaziantep University, TR-27310 GaZianteP, TuRKey

Email address: acikgoz@gantep.edu.tr ${ }^{2}$ 